\title{
Reserve Estimation from Early Time Production Data in Geopressured Gas Reservoir: Gas Production of Cumulative Unit Pressure Drop Method
}

\author{
Hongfeng Wang, ${ }^{1,2}$ Xiaoping Li, ${ }^{1}$ Hedong Sun $\mathbb{D}^{\mathrm{D}},{ }^{3}$ Guangren Meng, ${ }^{3}$ Wen Cao, ${ }^{3}$ \\ and Songbai $\mathrm{Zhu}^{2}$ \\ ${ }^{1}$ State Key Laboratory of Oil \& Gas Reservoir Geology and Exploitation (Southwest Petroleum University), China \\ ${ }^{2}$ PetroChina Tarim Oilfield Company, China \\ ${ }^{3}$ PetroChina Research Institute of Petroleum Exploration and Development, China
}

Correspondence should be addressed to Hedong Sun; sunhed@petrochina.com.cn

Received 8 March 2021; Accepted 27 April 2021; Published 1 June 2021

Academic Editor: Tianran Ma

Copyright (c) 2021 Hongfeng Wang et al. This is an open access article distributed under the Creative Commons Attribution License, which permits unrestricted use, distribution, and reproduction in any medium, provided the original work is properly cited.

\begin{abstract}
There is high uncertainty in reserve estimation during the early development of deep ultrahigh pressure gas reservoirs, largely because it remains challenging in accurately determining the formation compressibility. To overcome this, starting from the definition of compressibility, a novel gas production of cumulative unit pressure drop analysis method was established, of which the effectiveness was proven by applications in calculating the reserves of three gas reservoirs. It has been found that, in the limiting case, i.e., when the formation pressure dropped to the normal atmospheric pressure, the dimensionless gas production of the cumulative unit pressure drop was the reciprocal of the initial formation pressure. Besides, the relationship curve of the dimensionless gas production of the cumulative unit pressure drop and pressure drop was a straight line in the medium term, extending the straight line and intersecting the vertical line passing through the original formation pressure point, and the reserves can be determined according to the intersection point and the initial formation pressure. However, due to the influence of natural gas properties, the value needs further correction, and the correction coefficient depends on the pseudocritical temperature of natural gas. Specifically, when the pseudocritical temperature is given, the correction coefficient would be close to the minimum value of the natural gas deviation factor. When the pseudocritical temperature is more than 1.9 and less than 3.0, the minimum deviation factor would be between 0.90 and 1.0, and the higher the pseudocritical temperature, the closer the ratio is to 1.0 .
\end{abstract}

\section{Introduction}

The proportion of natural gas in primary energy consumption has been continuously increasing since the beginning of the $21^{\text {st }}$ century, demonstrating its importance as a global strategic resource and a livelihood material. According to existing reports [1], $61 \%$ of natural gas reserves which were discovered worldwide in the past decade are distributed in deep zones and are characterized by strong ground stress, high heterogeneity, temperature, and pressure. High pressure gas reservoirs have downward curving $p / Z \sim G_{\mathrm{p}}$ behavior. Precise estimation of the reserves of such gas reservoirs is crucial for reservoir engineers. However, reserve estimation's basic method is the material balance method [2]. The formation compressibility is one of the key parameters in the material balance equation of a high pressure gas reservoir but challenging to determine; the error in estimates could even exceed $100 \%$ [3]. For many years, the petroleum industry has relied on Hall's correlation [4] for estimating pore volume compressibility. This correlation was developed from 
TABLE 1: Published studies of reserve estimate problem of high pressure gas reservoir.

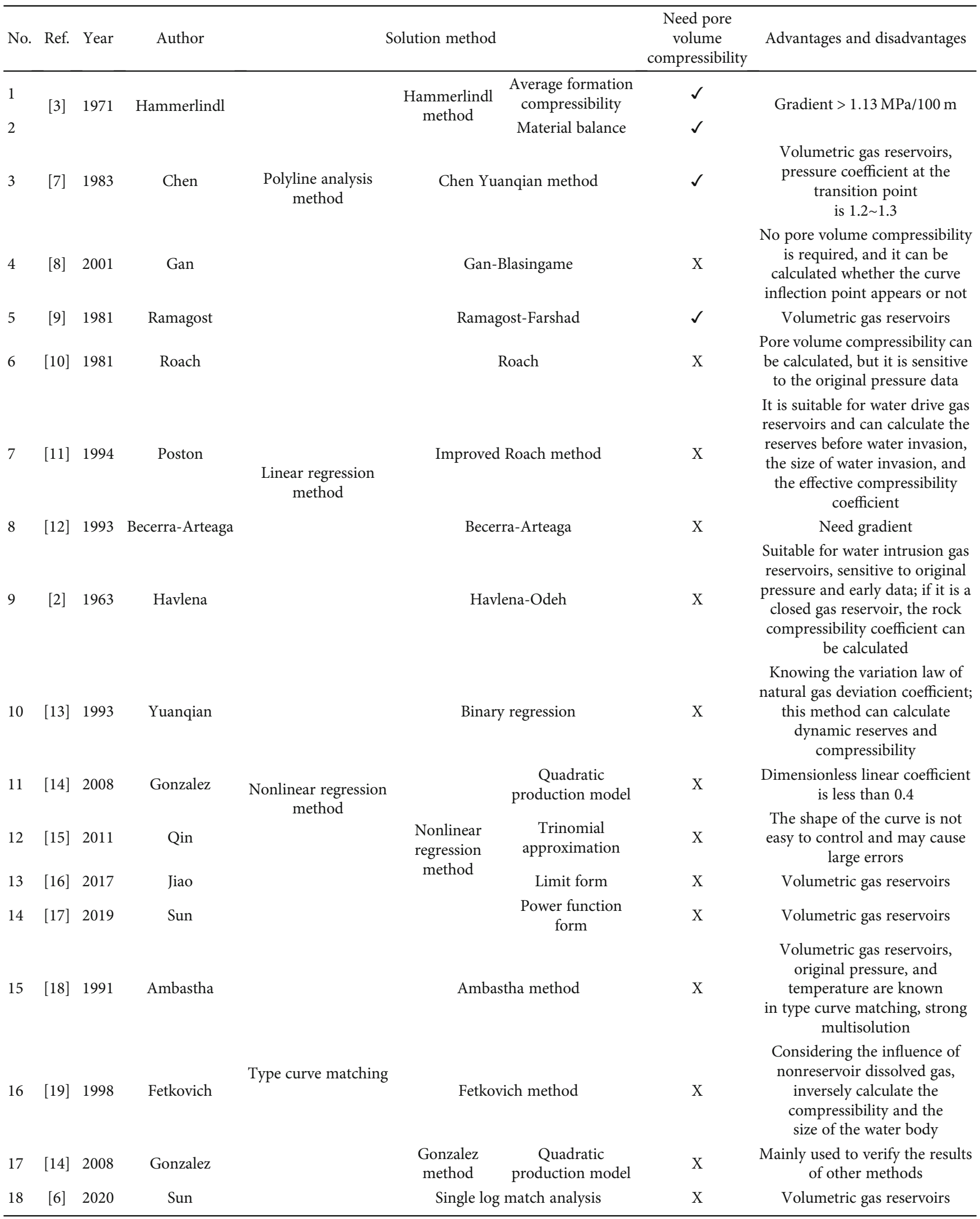


TABLE 1: Continued.

\begin{tabular}{cccccc}
\hline No. Ref. Year & Author & Solution method & $\begin{array}{c}\text { Need pore } \\
\text { volume } \\
\text { compressibility }\end{array}$ & $\begin{array}{c}\text { Advantages and disadvantages } \\
\text { of water invasion, only reserves } \\
\text { of connected well groups can } \\
\text { be calculated }\end{array}$ \\
\hline 19 & {$[20]$} & 2001 & Marhaendrajana & $\begin{array}{c}\text { Multiwell modern production } \\
\text { decline analysis method }\end{array}$ & $\begin{array}{c}\text { It can be used to calculate } \\
\text { the volume of water bodies and } \\
\text { calculate the water-soluble } \\
\text { gas reserves }\end{array}$ \\
\hline
\end{tabular}

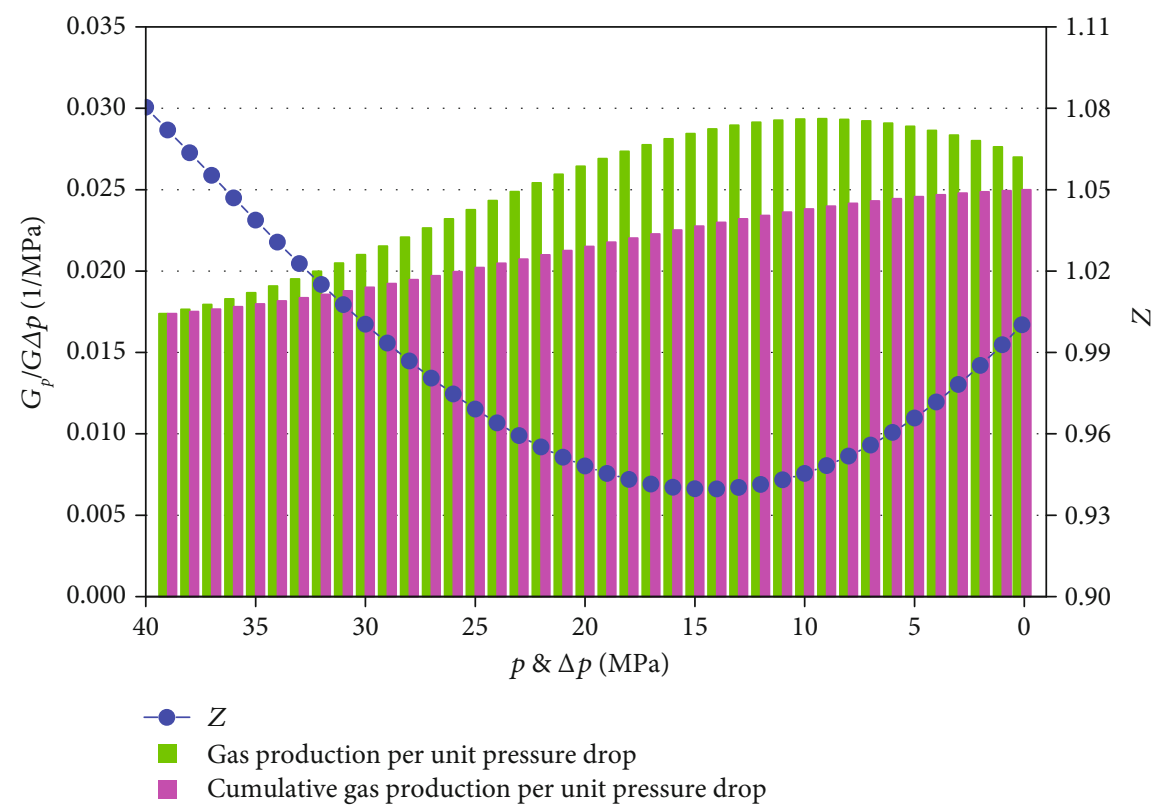

FIGURE 1: Gas production per unit pressure drop and dimensionless gas production of cumulative unit pressure drop (formation pressure is $40 \mathrm{MPa}$, formation temperature is $373.15 \mathrm{~K}$, and gravity of gas is 0.6 ).

measurements on seven consolidated limestone and five consolidated sandstone samples. Compressibilities, however, are highly affected by reservoir type and overburden conditions.

According to the classical theory, the material balance $p /$ $Z \sim G_{\mathrm{p}}$ curve of a high pressure gas reservoir has a twosegment characteristic [5], with the second straight line segment occurring late (apparent formation pressure drops by $6 \%-38 \%)$. However, for high pressure and ultrahigh pressure gas reservoirs at the early stage of development, even if the production test time is up to 1 year and the drawdown scope reaches $3 \%-38 \%$ of the initial formation pressure or even higher, the starting point deviating from the early straight line segment would still not occur, and thus, the starting condition of using the material balance method to calculate reserves cannot be met. If conditions are not fully met, arbitrary applications of this method will result in serious deviation in the calculation [6]. In recent years, tremendous efforts have been spent in designing methods independent of formation compressibility, including the type curve match analysis and nonlinear regression methods. Table 1 indicates the scope of various types of information on this subject, listing the relevant papers that have been published in the petroleum literature.
This has provided new solutions to the reserve estimate problem of a high pressure gas reservoir when the starting condition is met in the middle and late stages of development. However, a simple but practical method is still missing for the reserve estimate in the early development stage.

To bridge the gap, starting with the definition of formation compressibility and the material balance equation of volumetric gas reservoir, we established an analysis method for the reserve estimate based on gas production of cumulative unit pressure drop and evaluated its performance in three gas reservoirs.

\section{Gas Production of Cumulative Unit Pressure Drop}

2.1. Isothermal Compressibility of Gas. In general, the isothermal coefficient of compressibility of a material is defined as

$$
C_{\mathrm{f}}=-\frac{1}{V}\left(\frac{\partial V}{\partial p}\right)_{\mathrm{T}},
$$




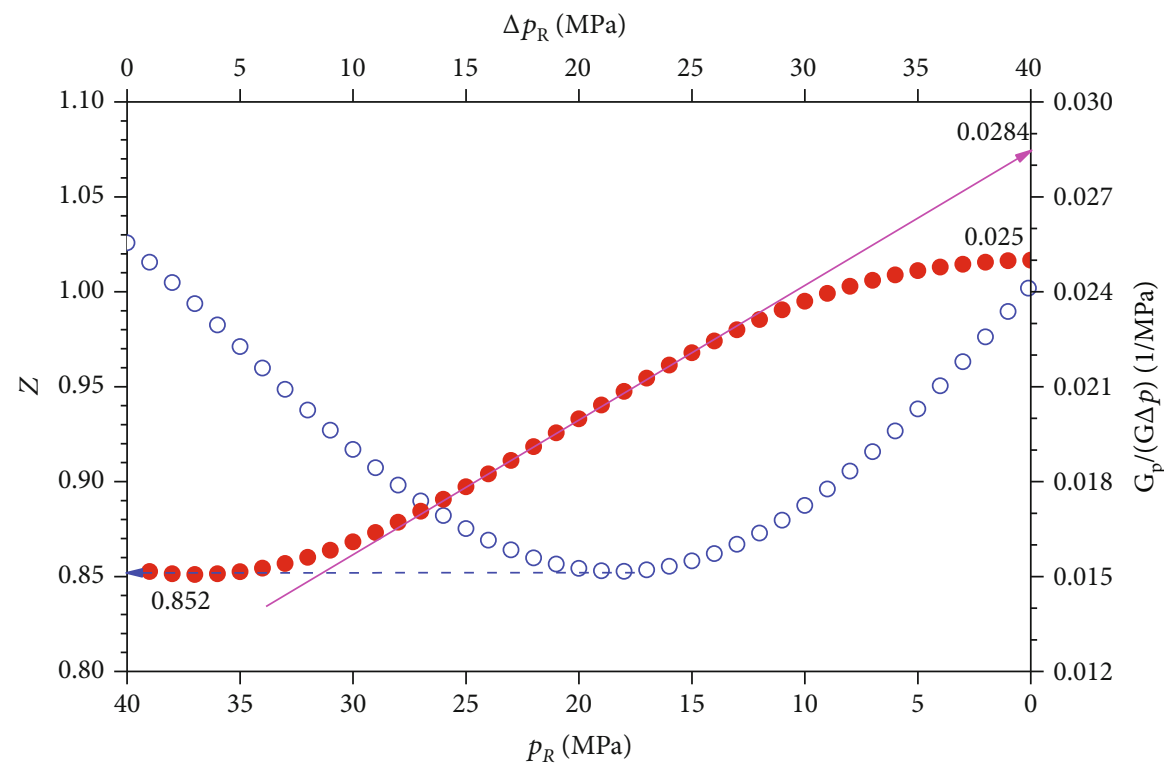

○ $Z$

- $G_{\mathrm{p}} /\left(G \Delta p_{\mathrm{R}}\right)$

(a) $333.15 \mathrm{~K}$

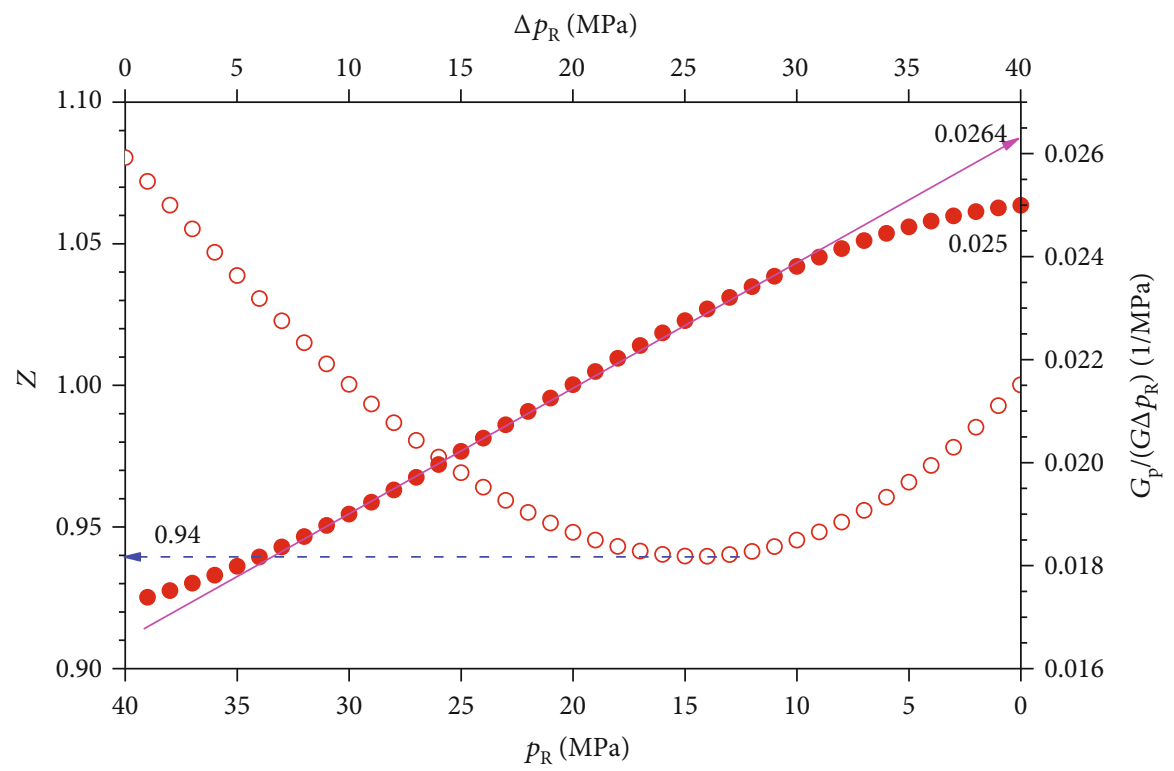

$\circ Z$

- $G_{\mathrm{p}} /\left(G \Delta p_{\mathrm{R}}\right)$

(b) $373.15 \mathrm{~K}$

FIgUre 2: Continued. 


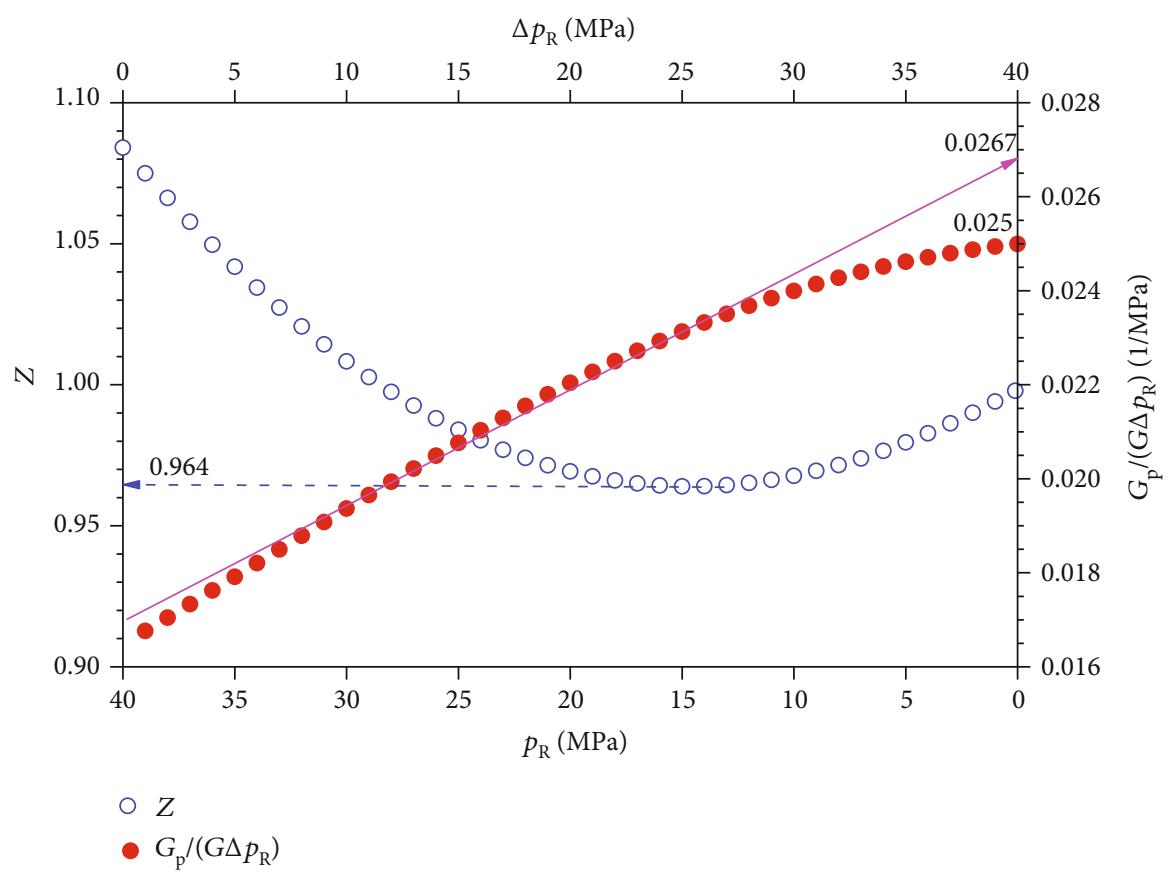

(c) $413.15 \mathrm{~K}$

FIGURE 2: Effect of temperature on gas production of cumulative pressure drop.

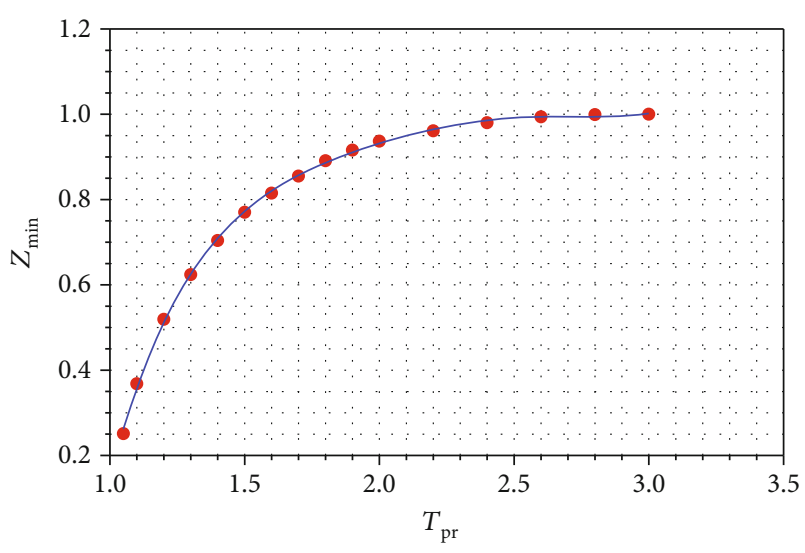

Figure 3: Minimum deviation factor at different pseudoreduced pressures.

where $C_{\mathrm{f}}$ denotes isothermal compressibility of gas in $1 / \mathrm{MPa}$, with $p$ denoting pressure in $\mathrm{MPa}$ and $V$ denoting volume in $10^{8} \mathrm{~m}^{3}$.

2.2. Gas Production of Cumulative Pressure Drop. Gas production per unit pressure drop is defined as cumulative gas production per unit drop of average formation pressure. Gas production of cumulative unit pressure drop is defined as the ratio of cumulative gas production to cumulative drawdown of formation pressure from the initial formation pressure condition to the current formation pressure condition.

When equation (1) is applied to a gas reservoir,

$$
C_{\mathrm{f}}=-\frac{1}{G}\left(\frac{\mathrm{d} G}{\mathrm{~d} p}\right)_{\mathrm{T}} \text {. }
$$

TABLE 2: Producing history of Anderson "L" gas reservoir (Duggan, 1972).

\begin{tabular}{lcccc}
\hline$p$ & $G_{\mathrm{p}}$ & $p / Z$ & $\Delta p$ & $G_{\mathrm{p}} / \Delta p$ \\
\hline $\mathrm{MPa}$ & $10^{8} \mathrm{~m}^{3}$ & $\mathrm{MPa}$ & $\mathrm{MPa}$ & $10^{8} \mathrm{~m}^{3} / \mathrm{MPa}$ \\
65.548 & 0.000 & 45.52 & 0 & 0.0000 \\
64.066 & 0.118 & 45.18 & 1.482 & 0.0796 \\
61.846 & 0.492 & 44.59 & 3.702 & 0.1329 \\
59.260 & 0.966 & 44.09 & 6.288 & 0.1536 \\
57.447 & 1.276 & 43.65 & 8.101 & 0.1575 \\
55.220 & 1.647 & 43.07 & 10.328 & 0.1595 \\
52.421 & 2.257 & 42.31 & 13.127 & 0.1719 \\
51.062 & 2.620 & 41.92 & 14.486 & 0.1809 \\
48.277 & 3.146 & 41.05 & 17.271 & 0.1822 \\
46.340 & 3.519 & 40.40 & 19.208 & 0.1832 \\
45.057 & 3.827 & 39.98 & 20.491 & 0.1868 \\
39.741 & 5.163 & 37.92 & 25.807 & 0.2001 \\
32.860 & 6.836 & 33.63 & 32.688 & 0.2091 \\
29.613 & 8.389 & 31.91 & 35.935 & 0.2334 \\
25.855 & 9.689 & 29.02 & 39.693 & 0.2441 \\
22.387 & 10.931 & 26.21 & 43.161 & 0.2533 \\
\hline
\end{tabular}

When separation variables are integrated,

$$
G_{\mathrm{p}}=G \int_{p}^{p_{\mathrm{i}}} C_{\mathrm{f}} \mathrm{d} p=\left[G \bar{C}_{-\mathrm{f}}\right]\left(p_{\mathrm{i}} p\right),
$$

where $\left[G \bar{C}_{-\mathrm{f}}\right]$ is the gas production of cumulative unit pressure drop, $10^{8} \mathrm{~m}^{3} / \mathrm{MPa}$. When the formation pressure drops to $0.101325 \mathrm{MPa}$, there is 


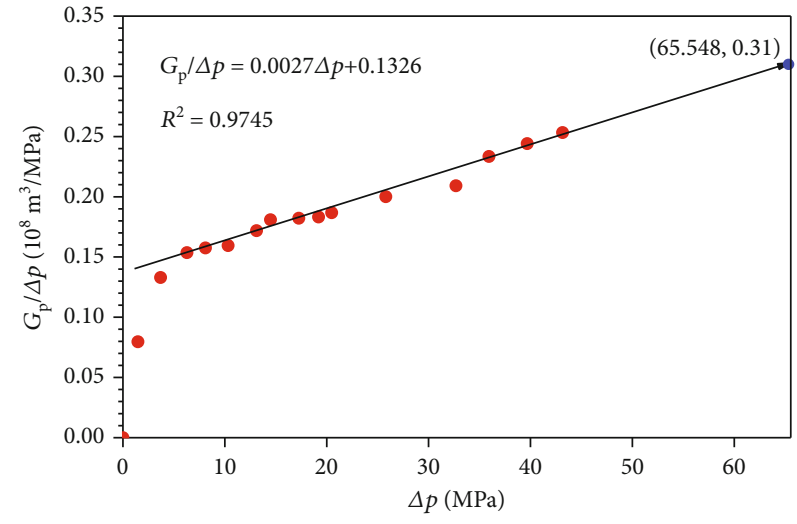

Figure 4: Reserves of Anderson "L" gas reservoir calculated by the method of gas production of cumulative unit pressure drop.

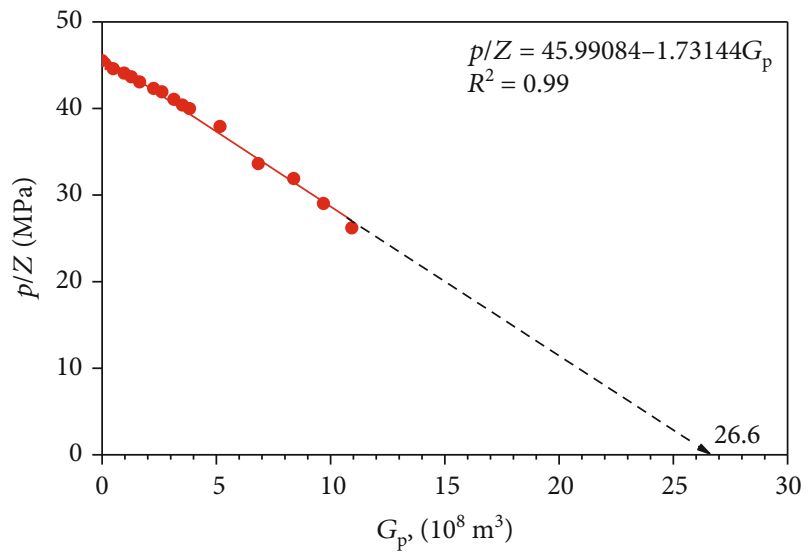

FIgURE 5: Reserves of Anderson "L" gas reservoir calculated by the p/Z curve method.

TABle 3: Producing history of Louisiana offshore gas reservoir (Ramagost, 1981).

\begin{tabular}{lcccc}
\hline$p$ & $G_{\mathrm{p}}$ & $p / Z$ & $\Delta p$ & $G_{\mathrm{p}} / \Delta p$ \\
\hline $\mathrm{MPa}$ & $10^{8} \mathrm{~m}^{3}$ & $\mathrm{MPa}$ & $\mathrm{MPa}$ & $10^{8} \mathrm{~m}^{3} / \mathrm{MPa}$ \\
78.903 & 0.000 & 52.743 & 0.000 & 0.0000 \\
73.595 & 2.809 & 51.178 & 5.309 & 0.5291 \\
69.851 & 8.104 & 50.000 & 9.053 & 0.8952 \\
63.797 & 15.178 & 47.968 & 15.106 & 1.0047 \\
59.116 & 21.994 & 46.184 & 19.788 & 1.1115 \\
54.510 & 28.719 & 44.317 & 24.394 & 1.1773 \\
50.883 & 34.082 & 42.687 & 28.020 & 1.2163 \\
47.208 & 41.062 & 40.908 & 31.695 & 1.2955 \\
44.044 & 45.485 & 39.255 & 34.860 & 1.3048 \\
40.176 & 51.633 & 37.062 & 38.728 & 1.3332 \\
37.294 & 55.991 & 35.283 & 41.610 & 1.3456 \\
34.474 & 61.068 & 33.372 & 44.430 & 1.3745 \\
31.026 & 66.754 & 30.872 & 47.877 & 1.3943 \\
28.751 & 69.631 & 29.100 & 50.152 & 1.3884 \\
\hline
\end{tabular}

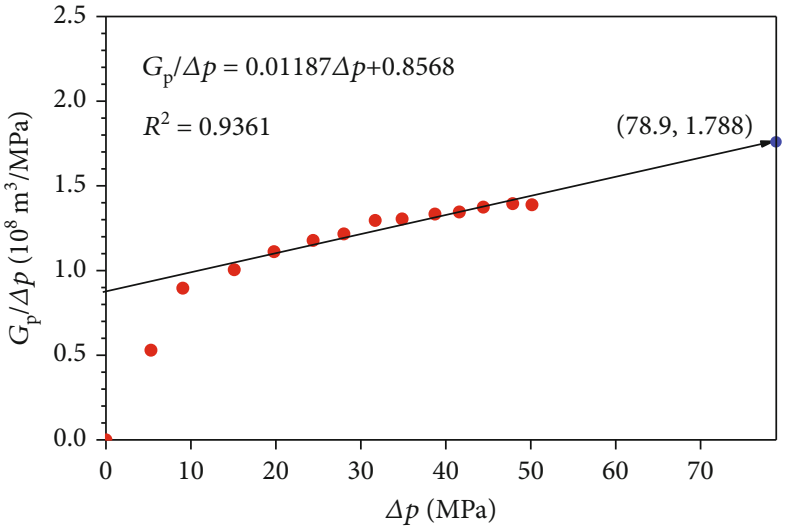

FIGURE 6: Reserves of Louisiana offshore gas reservoir calculated by the method of gas production of cumulative unit pressure drop.

$$
G_{\mathrm{p}}=G=\left[G \bar{C}_{-\mathrm{f}}\right] p_{\mathrm{i}}
$$

i.e., when the formation pressure drops to normal atmospheric pressure, the dimensionless gas production of the cumulative unit pressure drop is the reciprocal of the initial formation pressure.

2.3. Variation Law of Gas Production of Cumulative Unit Pressure Drop. The material balance equation of a volumetric gas reservoir can be expressed as

$$
\frac{p}{Z}=\left(\frac{p}{Z}\right)_{\mathrm{i}}\left(1-\frac{G_{\mathrm{p}}}{G}\right),
$$

where $p$ is the pressure, $\mathrm{MPa} ; G_{\mathrm{p}}$ is the cumulative gas production, $10^{8} \mathrm{~m}^{3}$; $G$ is the reserves, $10^{8} \mathrm{~m}^{3}$; and the subscript i represents the initial state. Equation (5) is deformed, and the dimensionless cumulative gas production is

$$
\frac{G_{\mathrm{p}}}{G}=1-\frac{p / Z}{(p / Z)_{\mathrm{i}}} .
$$

When the pressure drops to $p_{\mathrm{j}}$, the dimensionless cumulative gas production is

$$
\left(\frac{G_{\mathrm{p}}}{G}\right)_{\mathrm{j}}=1-\frac{(p / Z)_{\mathrm{j}}}{(p / Z)_{\mathrm{i}}} .
$$

When the pressure drops to $p_{\mathrm{j}+1}$, the dimensionless gas production is

$$
\left(\frac{G_{\mathrm{p}}}{G}\right)_{\mathrm{j}+1}=1-\frac{(p / Z)_{\mathrm{j}+1}}{(p / Z)_{\mathrm{i}}} .
$$

The dimensionless gas production per unit pressure drop (assuming $p_{\mathrm{j}}-p_{\mathrm{j}+1}=1$ ) is 


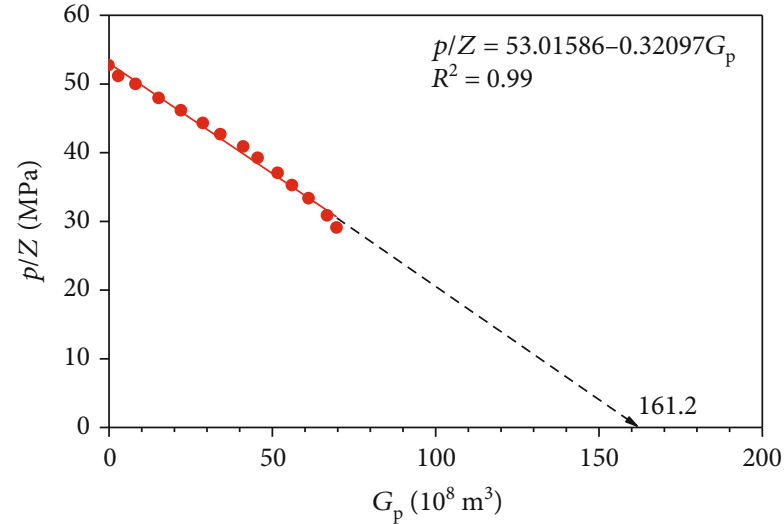

Figure 7: Reserves of Louisiana offshore gas reservoir calculated by the $p / Z$ curve method.

Table 4: Producing history of $\mathrm{X}$ gas reservoir.

\begin{tabular}{lcccc}
\hline$p$ & $G_{\mathrm{p}}$ & $p / Z$ & $\Delta p$ & $G_{\mathrm{p}} / \Delta p$ \\
\hline $\mathrm{MPa}$ & $10^{8} \mathrm{~m}^{3}$ & $\mathrm{MPa}$ & $\mathrm{MPa}$ & $10^{8} \mathrm{~m}^{3} / \mathrm{MPa}$ \\
74.48 & 0.00 & 53.06 & 0 & 0 \\
74.33 & 2.58 & 53.01 & 0.15 & 17.20 \\
72.67 & 34.96 & 52.42 & 1.81 & 19.31 \\
68.55 & 119.74 & 50.95 & 5.93 & 20.19 \\
63.53 & 226.48 & 49.13 & 10.95 & 20.68 \\
58.45 & 338.60 & 47.18 & 16.03 & 21.12 \\
53.84 & 451.04 & 45.25 & 20.64 & 21.85 \\
49.53 & 555.25 & 43.26 & 24.95 & 22.25 \\
45.70 & 659.46 & 41.29 & 28.78 & 22.91 \\
41.71 & 763.96 & 39.01 & 32.77 & 23.31 \\
38.06 & 868.17 & 36.69 & 36.42 & 23.84 \\
34.59 & 972.38 & 34.24 & 39.89 & 24.38 \\
31.01 & 1076.59 & 31.47 & 43.47 & 24.77 \\
27.83 & 1181.08 & 28.79 & 46.65 & 25.32 \\
24.73 & 1285.29 & 25.98 & 49.75 & 25.83 \\
21.52 & 1389.50 & 22.89 & 52.96 & 26.24 \\
18.46 & 1493.16 & 19.78 & 56.02 & 26.65 \\
15.55 & 1593.89 & 16.72 & 58.93 & 27.05 \\
\hline & & & &
\end{tabular}

$$
\left(\frac{G_{\mathrm{p}}}{G}\right)_{\mathrm{j}+1}-\left(\frac{G_{\mathrm{p}}}{G}\right)_{\mathrm{j}}=\frac{(p / Z)_{\mathrm{j}}}{(p / Z)_{\mathrm{i}}}-\frac{(p / Z)_{\mathrm{j}+1}}{(p / Z)_{\mathrm{i}}}
$$

The dimensionless gas production of cumulative unit pressure drop is

$$
\frac{\left(G_{\mathrm{p}} / G\right)_{\mathrm{j}}}{p_{\mathrm{i}}-p_{\mathrm{j}}}=\frac{1-(p / Z)_{\mathrm{j}} /(p / Z)_{\mathrm{i}}}{p_{\mathrm{i}}-p_{\mathrm{j}}}
$$

The theoretical calculation results show that with the depletion of formation pressure, affected by the deviation factor, the gas production per unit pressure drop first increases and then decreases, while the dimensionless gas production of cumulative unit pressure drop increases gradually. When

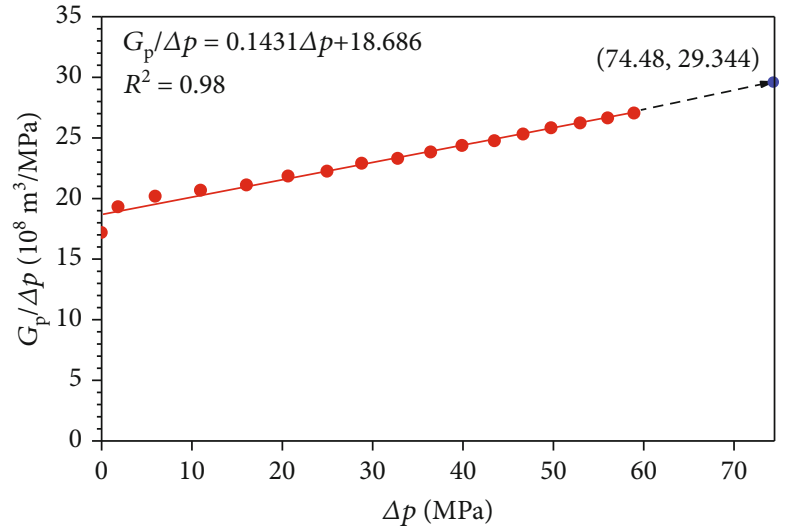

FIgURE 8: Reserves of $\mathrm{X}$ gas reservoir calculated by the method of gas production of cumulative unit pressure drop.

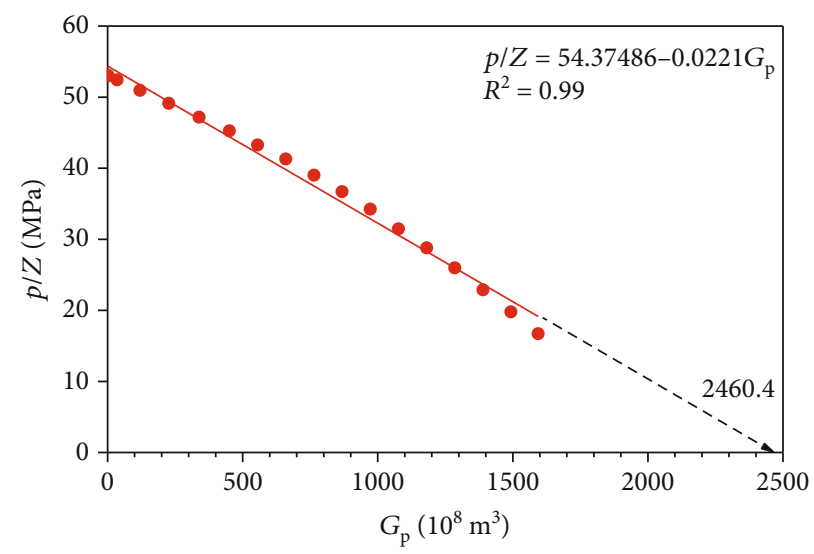

FIgURE 9: Reserves of $X$ gas reservoir calculated by the $p / Z$ method.

the formation pressure drops to atmospheric pressure, its value is the reciprocal of the initial formation pressure, as shown in Figure 1.

Figure 2 shows the variation of gas production of cumulative unit pressure drop with the drop of formation pressure at different temperatures, when the formation pressure is $40 \mathrm{MPa}$ and the gravity of gas is 0.6 . The relation curve of gas production of cumulative unit pressure drop and cumulative pressure drop is linear at early and middle stages; when the linear relation is prolonged to intersect with the vertical axis, the ratio $a$ of gas production of cumulative unit pressure drop to intersection value is approximately equal to the minimum deviation factor. In Figure 2(a), the temperature is $333.15 \mathrm{~K}, a=0.025 / 0.0284=0.88 \approx 0.85$, and the relative error is $3.6 \%$; in Figure $2(\mathrm{~b})$, the temperature is $373.15 \mathrm{~K}$, $a=0.025 / 0.0264=0.95 \approx 0.94$, and the relative error is $1.1 \%$; in Figure 2(c), the temperature is $413.15 \mathrm{~K}, a=0.025$ / $0.0267=0.94 \approx 0.96$, and the relative error is $2.1 \%$.

2.4. Minimum Deviation Factor of Natural Gas. The minimum deviation factor of natural gas depends on the pseudocritical property [22], as shown in Figure 3. When the pseudoreduced temperature is more than 1.9 , the minimum deviation factor of natural gas is above 0.90 . 
TABLE 5: Comparison of reserves calculated by different methods.

\begin{tabular}{lccccc}
\hline Gas reservoir & $\begin{array}{c}\text { Volumetric method } \\
\text { reserves }\left(10^{8} \mathrm{~m}^{3}\right)\end{array}$ & $p / Z$ curve method & Nonlinear regression method & $\begin{array}{c}\text { Reserves }\left(10^{8} \mathrm{~m}^{3}\right) \\
\text { match method type curve }\end{array}$ & $\begin{array}{c}\text { Method introduced } \\
\text { in the paper }\end{array}$ \\
\hline Anderson "L" & 19.68 & 26.6 & 19.9 & 20.0 & 19.0 \\
Louisiana offshore & 131.6 & 161.2 & 125.3 & 130.0 & 131.2 \\
$\mathrm{X}$ & 2091.5 & 2460.4 & 2032.0 & 2000.0 & 2032.55 \\
\hline
\end{tabular}

When the pseudoreduced temperature is unknown, it can be calculated based on the gravity of natural gas, as given by

$$
T_{\mathrm{pc}}=\frac{168+325 \gamma_{\mathrm{g}}-12.5 \gamma_{\mathrm{g}}^{2}}{1.8} .
$$

2.5. Calculation Method of Reserves. To sum up, the Cartesian plot of gas production of the cumulative unit pressure drop and the cumulative pressure drop is drawn, the linear regression is carried out, the straight line is prolonged to intersect with the vertical axis (as shown in Figure 2), and the product of the intersection ordinate value and the minimum deviation factor gives the reserve.

\section{Case Analysis}

3.1. Small Ultrahigh Pressure Gas Reservoir: Anderson "L" Gas Reservoir. The basic parameters of the American Anderson "L" gas reservoir [23] are as follows: the buried depth is $3404.5 \mathrm{~m}$, the pressure coefficient is $1.907 \mathrm{MPa} / 100 \mathrm{~m}$, the initial pressure is $65.55 \mathrm{MPa}$, the temperature is $403 \mathrm{~K}$, the gravity of natural gas is 0.656 , and the volumetric reserves are 19.68 $\times 10^{8} \mathrm{~m}^{3}$, with the producing history listed as in Table 2 .

The relation curve of the cumulative pressure drop and the gas production of the cumulative unit pressure drop is given in Figure 4; linear regression is carried out for the data points, the straight line is prolonged to intersect with the vertical axis, and the intersection ordinate value is $0.31 \times 10^{8}$ $\mathrm{m}^{3} / \mathrm{MPa}$, which is multiplied by the initial formation pressure $65.548 \mathrm{MPa}$, and the reserve is obtained as $20.29 \times 10^{8}$ $\mathrm{m}^{3}$. The minimum deviation factor is 0.935 based on Figure 3. Therefore, the reserves of the gas reservoir is estimated as $20.29 \times 0.935$, i.e., $19.00 \times 10^{8} \mathrm{~m}^{3}$. The reserves calculated by this method are $3.5 \%$ smaller than that by the volumetric method. The $p / Z$ curve method, which gave an estimation of $26.6 \times 10^{8} \mathrm{~m}^{3}$, is shown in Figure 5 .

3.2. Medium Ultrahigh Pressure Gas Reservoir: Louisiana Offshore Gas Reservoir. The basic parameters of the American Louisiana offshore ultrahigh pressure gas reservoir [23] are as follows: the buried depth is $4055 \mathrm{~m}$, the pressure coefficient is $1.95 \mathrm{MPa} / 100 \mathrm{~m}$, the initial pressure is $78.903 \mathrm{MPa}$, the temperature is $401.55 \mathrm{~K}$, the gravity of the natural gas is 0.60 , the pseudoreduced temperature is 1.96 , the initial water saturation is 0.22 , and the reserves are $131.6 \times 10^{8} \mathrm{~m}^{3}$, with the producing history listed as in Table 3.

The relation curve of the cumulative pressure drop and gas production of the cumulative unit pressure drop is given in Figure 6. The intersection ordinate value is $1.788 \times 10^{8}$ $\mathrm{m}^{3} / \mathrm{MPa}$, which is multiplied by the initial formation pressure $78.903 \mathrm{MPa}$ leading to the reserves of $141.07 \times 10^{8} \mathrm{~m}^{3}$. The pseudoreduced temperature of the gas reservoir is 1.96 , and the minimum deviation factor is 0.93 . Therefore, the reserve of the gas reservoir is $141.07 \times 0.93$, i.e., $131.2 \times 10^{8}$ $\mathrm{m}^{3}$. The reserves calculated by this method are highly consistent with that calculated by the dynamic method in the references [9]. If the reserves are calculated directly by the $p / Z$ curve method, the calculated results are $161.2 \times 10^{8} \mathrm{~m}^{3}$, as shown in Figure 7, which are much larger than the values calculated by the new method.

3.3. Large High Pressure Gas Reservoir: X Gas Reservoir. The basic parameters of the $X$ gas reservoir [16] are as follows: the middle buried depth is $3750 \mathrm{~m}$, the pressure coefficient is 2.0 $\mathrm{MPa} / 100 \mathrm{~m}$, the initial formation pressure is $74.48 \mathrm{MPa}$, the minimum deviation factor is 0.93 , and the reserves calculated by the volumetric method are $2091.5 \times 10^{8} \mathrm{~m}^{3}$, with the producing history listed as in Table 4.

The relation curve of the cumulative pressure drop and gas production of the cumulative unit pressure drop is given in Figure 8. The intersection ordinate value is $29.344 \times 10^{8}$ $\mathrm{m}^{3} / \mathrm{MPa}$, which is multiplied by the initial formation pressure $74.48 \mathrm{MPa}$ leading to the reserves of $2185.54 \times 10^{8} \mathrm{~m}^{3}$. The minimum deviation factor is 0.93 . Therefore, the reserve of the gas reservoir is $2185.54 \times 0.93$, i.e., $2032.55 \times 10^{8} \mathrm{~m}^{3}$, which is $2.8 \%$ lower than the reserves calculated by the volumetric method. If the reserves are calculated directly by the $\mathrm{p} / \mathrm{Z}$ method, the calculated result is $2460.4 \times 10^{8} \mathrm{~m}^{3}$, as shown in Figure 9, which is $17.6 \%$ higher than the reserves calculated by the static method.

3.4. Comparison with Calculated Results of Other Methods without Considering Formation Compressibility. As seen in Table 5, the calculated results of the method introduced in the paper are close to those of the nonlinear regression method and the semilogarithmic type curve match method, and the three cases further verify the correctness of the method. When the gas reservoir is in the early stage of development and there is no obvious inflection point in the $p / Z$ curve, this method can be used to approximately estimate the reserves of the gas reservoir.

\section{Conclusions and Suggestions}

(1) Since it is difficult to accurately determine the formation compressibility in the material balance equation of high pressure gas reservoirs, the method without 
considering the formation compressibility is recommended for reserve estimation

(2) When the formation pressure drops to the normal atmospheric pressure, the dimensionless gas production of the cumulative unit pressure drop is the reciprocal of the initial formation pressure. The relation curve of the dimensionless gas production of the cumulative unit pressure drop and pressure drop is a straight line in the medium term. The reserve correction coefficient is related to temperature

(3) The method of gas production of cumulative unit pressure drop, the semilog curve match analysis technique, and the nonlinear regression method can be used in turn to calculate the reserves based on the length of production time. If the production time is short, the reserves can be roughly estimated by the method of gas production of the cumulative unit pressure drop. Based on the reserve estimate results, combined with material balance equation, the effective compressibility coefficient can be calculated, and then, the water influx can be calculated

(4) For the water drive gas reservoir, the reserves calculated by the method of gas production of the cumulative unit pressure drop are relatively optimistic; in the future, the evaluation method of water drive gas reservoirs should be further studied to reduce the ambiguity of analysis results

\section{Data Availability}

Previously reported data were used to support this study and are available at [doi:10.2118/10125-MS; doi:10.1016/j .ijhydene.2017.04.190; doi:10.2118/2938-PA]. These prior studies (and datasets) are cited at relevant places within the text as references $[9,16,23]$.

\section{Conflicts of Interest}

The authors declare that there are no conflicts of interest regarding the publication of this paper.

\section{Acknowledgments}

This work is financially supported by the project of the Major Science and Technology Project of PetroChina Company Limited "Research and Application of Key Technologies for Development of Deep and Ultra-Deep Gas Fields in Kuqa Depression" (No. 2018E-1803).

\section{References}

[1] Z. Zhe, "Analysis of global oil and gas exploration characteristics during the past decade," Oil Forum, vol. 38, no. 3, pp. 5864, 2019.

[2] D. Havlena and A. S. Odeh, "The material balance as an equation of a straight line," Journal of Petroleum Technology, vol. 15 , no. 8, pp. 896-900, 1963.
[3] D. J. Hammerlindl, "Predicting gas reserves in abnormally pressured reservoirs," in Fall Meeting of the Society of Petroleum Engineers of AIME, pp. 1-8, New Orleans, LA, USA, October 1971.

[4] H. N. Hall, "Compressibility of reservoir rocks," Journal of Petroleum Technology, vol. 5, no. 1, pp. 17-19, 1953.

[5] B. Craft, M. Hawkins, and R. Terry, Applied Petroleum Reservoir Engineering, Prentice Hall, 2nd edition, 1990.

[6] H. Sun, W. Cao, J. Li et al., "A material balance based practical analysis method to improve the dynamic reserve evaluation reliability of ultra-deep gas reservoirs with ultra-high pressure," Natural Gas Industry B, vol. 8, no. 1, pp. 79-87, 2021.

[7] C. Yuanqian, "Application and derivation of material balance equation for abnormally pressured gas reservoirs," Acta Petrolei Sinica, vol. 4, no. 1, pp. 45-53, 1983.

[8] R. G. Gan and T. A. Blasingame, "A semianalytical p/Z technique for the analysis of reservoir performance from abnormally pressured gas reservoirs," in SPE Annual Technical Conference and Exhibitionpp. 1-15, New Orleans, Louisiana, 2001.

[9] B. P. Ramagost and F. F. Farshad, "P/Z abnormally pressured gas reservoirs," in SPE Annual Technical Conference and Exhibition, pp. 1-10, 1981.

[10] R. H. Roach, "Analyzing geopressured reservoirs - a material balance technique," in SPE 9968-Unsolicated, pp. 1-4, 1981.

[11] S. W. Poston, H. Y. Chen, and M. J. Akhtar, "Differentiating formation compressibility and water-influx effects in overpressured gas reservoirs," SPE Reservoir Engineering, vol. 9, no. 3, pp. 183-187, 1994.

[12] Becerra-Arteaga, Analysis of Abnormally Pressured Gas Reservoirs, Texas A \& M University, 1993.

[13] C. Yuanqian and J. Hu, "A new method for determining gas in place and effective compressibility in abnormal high pressure reservoir," Natural Gas Industry, vol. 13, no. 1, pp. 5358, 1993.

[14] F. E. Gonzalez, D. Ilk, and T. A. Blasingame, "A quadratic cumulative production model for the material balance of an abnormally pressured gas reservoir," in SPE Western Regional and Pacific Section AAPG Joint Meeting, pp. 1-33, 2008.

[15] Z. Qin and L. Zhibin, "A new material balance model of an abnormally pressured gas reservoirs which contains cubic term of cumulative production and its calculation," Acta Scientiarum Naturalium Universitatis Pekinensis, vol. 47, no. 1, pp. 115-119, 2011.

[16] Y. Jiao, J. Xia, P. Liu et al., "New material balance analysis method for abnormally high-pressured gas-hydrocarbon reservoir with water influx," International Journal of Hydrogen Energy, vol. 42, no. 29, pp. 18718-18727, 2017.

[17] H. Sun, H. Wang, S. Zhu et al., "Reserve evaluation of high pressure and ultra-high pressure reservoirs with power function material balance method," Natural Gas Industry B, vol. 6, no. 5, pp. 509-516, 2019.

[18] A. K. Ambastha, "A type curve matching procedure for material balance analysis of production data from geopressured gas reservoirs," Journal of Canadian Petroleum Technology, vol. 30, no. 5, pp. 61-65, 1991.

[19] M. J. Fetkovich, D. E. Reese, and C. H. Whitson, "Application of a general material balance for high-pressure gas reservoirs (includes associated paper 51360)," SPE Journal, vol. 3, no. 1, pp. 3-13, 1998. 
[20] T. Marhaendrajana and T. A. Blasingame, "Decline curve analysis using type curves - evaluation of well performance behavior in a multiwell reservoir system," SPE Annual Technical Conference and Exhibition, 2001, pp. 1-15, New Orleans, Louisiana, 2001.

[21] M. P. Walsh, "Discussion of application of material balance for high pressure gas reservoirs," SPE Journal, vol. 3, no. 1, pp. 402-404, 1998.

[22] M. B. Standing, Volumetric and Phase Behavior of Oilfield Hydrocarbon Systems, Society of Petroleum Engineers of AIME, Richardson, TX, USA, 1977.

[23] J. O. Duggan, "The Anderson 'L'-an abnormally pressured gas reservoir in South Texas," Journal of Petroleum Technology, vol. 24, no. 2, pp. 132-138, 1972. 